\title{
Data on the Populations of Bombina variegata (Amphibia: Anura: Bombinatoridae) from Cozia National Park and Its Surrounding Areas (Vâlcea County, Romania)
}

\author{
Alexandru IFTIME ${ }^{1, *}$, Oana IFTIME2 \\ 1"Grigore Antipa” National Museum of Natural History, Şos. Kiseleff 1, 011341 Bucharest 1, Romania \\ ${ }^{2}$ Department of Genetics, Faculty of Biology, University of Bucharest, Aleea Portocalelor 1-3, 060101 \\ Bucharest 6, Romania \\ *corresponding author, e-mail: aiftime@antipa.ro
}

Received: November 11, 2016; Accepted: May 17, 2017; Available online: June 29, 2017; Printed: June 30, 2017

\begin{abstract}
The results of surveys upon the Bombina variegata populations in the Cozia National Park are presented. 52 location points were found. The species is widespread in the investigated area, is resilient to some extent to the current level of natural and human disturbance, is moderately anthropophilic and shows evidence of previous introgressive hybridization with $B$. bombina. The habitats of the various populations in the area show different quality, connectivity and dynamics. These data should be useful for any management strategy for Bombina variegata in the National Park and its surrounding areas.
\end{abstract}

Key words. Cozia National Park, Bombina variegata, populations, distribution, habitat, dynamics, introgressive hybridization.

\section{INTRODUCTION}

The Cozia National Park is a part of both the National Park network and the Natura 2000 network of Romania. As a Natura 2000 Site of Community Importance, the Cozia National Park is dedicated to the protection via conservation-orriented management of a number of Natura 2000 species (species on the Annex II of the 92/43/EEC Directive), among which the Yellow-Bellied Toad, Bombina variegata. This species is widely distributed in Europe (Speybroeck et al., 2016) and Romania, still locally common in many montane and submontane areas of Romania (Cogălniceanu et al., 2000; Cogălniceanu et al., 2013), but also declining in other areas (Cogălniceanu et al., 2000; Iftime, 2005). Good knowledge of the population particulars for a species within a protected area is necessary for its management, including distribution, habitat requirements and characteristics, metapopulational dynamics, population connectivity, hybridization and the reaction to human and natural changes in its habitat. We try to improve upon the previous knowledge of Bombina variegata in the Cozia National Park (researched herpetologically by us in the recent past - Iftime \& Iftime, 2006, 2007) by highlighting these aspects.

\section{MATERIAL AND METHODS}

This paper is based upon field work performed in September 2014, May and July 2015 and May 2016, mostly within the grant PN-II-PT-PCCA-2013-4-1489, but also making use of independently obtained data. Qualitative observations were undertaken using the active transects method (after McDiarmid, 1992, and Heyer et al., 1994, in Cogălniceanu, 1997), the transect being $4 \mathrm{~m}$ wide. The geographical 
coordinates for all locations were acquired by GPS. On some transects (Valea lui Stan, Spinu, Lotrișor-Narâțu, Păușa, Căciulata, Olt valley, Lotrișor-Căpățânii) photographs of the ventral pattern were taken for a number of specimens, ensuring recognition for sampling studies within the same project. The morphological Bombina variegata-like vs. Bombina bombina-like character states were analyzed in order to evidence possible past introgressive hybridization using character tables (Tabs 1,2), each containing a set of 10 characters - after Gollmann, 1984; Szymura \& Barton, 1991; Stugren, 1980; Ghira \& Mara, 2000; Covaciu-Marcov et al., 2003; Sas et al., 2005, modified by us (Iftime \& Iftime, 2007 - Tabs 1, 2). Each Bombina bombina-like character state is counted as 0 and each $B$. variegata-like as 1 ; thus for each set of characters a score is obtained for each specimen. The average of these two scores is obtained for each specimen, ranging between 0 ("pure" B. bombina) and 10 ("pure" B. variegata). The median average, maximum and minimum values and quartiles are calculated for each population from the scores of pertaining specimens.

\section{RESULTS}

We found Bombina variegata in 52 points in and around the Cozia National Park. The distribution points, with their localities (grouped by major topographic feature: i.e. valley or another area of contiguity, that empirically separate local [sub] populations), visit year(s), GPS coordinates, and data pertaining to reproduction (whether it was observed at a given point), altitude and ecological characteristics (altitude, habitat type, persistence of habitat) are given in table 3; a map showing the points is given in fig. 1 .

The average character score for each locality/population investigated in this respect is given in table 4 .

\section{DISCUSSION}

Our results show that Bombina variegata has a wide distribution in and around Cozia National Park (Fig. 1). However, as can be easily observed (Figs 1, 2), the populations of this species are mostly found at relatively low altitudes (350-650

Table 1

First set of characters and states used to determine a score reflecting introgressive hybridization in Bombina species.

\begin{tabular}{|l|l|l|}
\hline Character & Bombina bombina & Bombina variegata \\
\hline Light ventral coloration & Red, orange, deep yellow & Yellow, light yellow \\
\hline $\begin{array}{l}\text { Colour of the upper part of the first } \\
\text { finger and of the finger tips }\end{array}$ & Black & Yellow \\
\hline Dorsal coloration & Greenish-grey & Brownish-grey \\
\hline Tarsal and palmar light spots, to each other & Separated & United \\
\hline Ventral coloration & Light spots on a dark ground & Dark spots on a light ground \\
\hline Relation of head length and width & Length $>$ width & Length $<$ width \\
\hline Ventral and lateral warts & White dots around warts & No white dots around warts \\
\hline Dorsal dark spots & Symmetrical & Irregular \\
\hline Dorsal warts & Blunt & Sharp, giving rugous aspect \\
\hline $\begin{array}{l}\text { Raport of tibio-tarsal articulations when } \\
\text { stylopode and zeugopode are parallel }\end{array}$ & Not touching & Touching \\
\hline
\end{tabular}


Second set of characters and states used to determine a score reflecting introgressive hybridization inBombina species.

\begin{tabular}{|l|l|l|}
\hline Character (Light spots on the...) & Bombina bombina & Bombina variegata \\
\hline Throat-throat & Separated & United \\
\hline Throat-chest & Separated & United \\
\hline Chest-chest & Separated & United \\
\hline Chest-shoulder & Separated & United \\
\hline Shoulder-arm & Separated & United \\
\hline Chest-abdomen & Separated & United \\
\hline Abdomen-abdomen & Separated & United \\
\hline Abdomen-basin & Separated & United \\
\hline Basin-basin & Separated & United \\
\hline Basin-thigh & Separated & United \\
\hline
\end{tabular}

$\mathrm{m}$ a.s.1.), a rare occurrence at $1115 \mathrm{~m}$ a.s.1. breaking this pattern - while in many other Romanian montane areas Bombina variegata is found commonly at much higher altitudes (Iftime \& Iftime, 2014). This can be explained by the mostly unhospitable nature of the upper reaches of the Cozia and surrounding mountains: very steep, rocky areas that are either quite dry and exposed or washed by very quick-flowing streams that do not afford suitable habitat for Bombina variegata. This situation has the double effect of confining Bombina variegata to the lower areas and creating discrete valley populations, more or less effectively separated by intervening ridges (some marginal populations, such as those from Spinu and the Pripoare-Dângești area, do not fit into this pattern and are definitely not beyond linking to each other, as the terrain there is a series of rolling foothills with slow-flowing rivulets). However, where conditions are proper Bombina variegata can and does occur higher up and even breeds there (e.g. at Stânișoara Monastery, 736 m a.s.1.).

The habitat types are in relation to the above-mentioned aspect. Most of them are road-side ditches, stream-side ponds being also quite numerous (Fig. 3); obviously small stagnant waterbodies are the most favourable habitats. Streams and small rivers are also good enough, provided their flow is not much too strong. Very steep angles of slopes are unfavourable for either of the above. We were, however, unable to observe any Bombina in the large, slow-flowing, dammed Olt River; it seems to be avoided, for toads living in its proximity, in small-river habitats contiguous to the Olt dam lakes, never appeared to venture in these last.

The metapopulational dynamic is also evident. Of the habitats supporting Bombina variegata that were investigated over more than one year, less than a quarter (Fig. 4) were not persistent over the whole investigation period. The causes are both natural and artificial (Tab. 3). Some water points dry up despite not especially dry years; others form anew, and others are colonized anew by Bombina variegata. Also, major floods (such as occurred in 2014) seem to have a tremendous impact: after the torrential flooding, in September 2014, the Băiașu valley was apparently devoid of Bombina variegata and the habitats were washed downstream. In 2015 a single element of the presence of Bombina variegata was found - a dead specimen - and in 2016 a breeding population was formed in a place where in 2014 and 2015 there was 
Locations investigated with their geographical coordinates and characteristics. Data on species'reproduction.

\begin{tabular}{|c|c|c|c|}
\hline $\begin{array}{l}\text { Locality/population, } \\
\text { visit year(s) }\end{array}$ & $\begin{array}{l}\text { GPS } \\
\text { Coordinates }\end{array}$ & Reproduction & Altitude and ecological characteristics \\
\hline \multirow[t]{2}{*}{ Valea lui Stan 2014-16 } & $\begin{array}{l}\text { N45 } 20.100 \\
\text { E24 } 11.836 \\
\end{array}$ & $\begin{array}{l}\text { Yes (eggs, larvae and/ } \\
\text { or metamorphs present) }\end{array}$ & $\begin{array}{l}354 \mathrm{~m} \text {; ponds on stream side; mixed } \\
\text { deciduous forest and grassy clearing }\end{array}$ \\
\hline & $\begin{array}{l}\text { N45 } 20.227 \\
\text { E24 } 11.796\end{array}$ & $\begin{array}{l}\text { Inaparent (adults and/ } \\
\text { or subadults only) }\end{array}$ & $\begin{array}{l}431 \mathrm{~m} \text {; ponds on stream side; mixed } \\
\text { deciduous forest and grassy clearing }\end{array}$ \\
\hline Spinu 2014-16 & $\begin{array}{l}\text { N45 } 22.682 \\
\text { E24 24.061 } \\
\end{array}$ & $\begin{array}{l}\text { Yes (eggs, larvae and/ } \\
\text { or metamorphs present) }\end{array}$ & $527 \mathrm{~m}$; slow-flowing stream; rural mosaic \\
\hline \multirow[t]{6}{*}{$\begin{array}{l}\text { Lotrișor-Narâțu } \\
2014-16\end{array}$} & $\begin{array}{ll}\text { N45 } & 18.227 \\
\text { E24 } & 16.970 \\
\end{array}$ & $\begin{array}{l}\text { Yes (eggs, larvae and/ } \\
\text { or metamorphs present) }\end{array}$ & $271 \mathrm{~m}$; ponds on river side; mixed deciduous forest \\
\hline & $\begin{array}{l}\text { N45 } 17.277 \\
\text { E24 } 14.140\end{array}$ & $\begin{array}{l}\text { Yes (eggs, larvae and/ } \\
\text { or metamorphs present) }\end{array}$ & $\begin{array}{l}661 \mathrm{~m} \text {; road-side ditch; beech and } \\
\text { mixed forest; dried } 2016\end{array}$ \\
\hline & $\begin{array}{l}\text { N45 } 18.188 \\
\text { E24 } 16.963\end{array}$ & $\begin{array}{l}\text { Inaparent (adults and/ } \\
\text { or subadults only) }\end{array}$ & $309 \mathrm{~m}$; ponds on river side; mixed deciduous forest \\
\hline & $\begin{array}{l}\text { N45 } 17.566 \\
\text { E24 } 14.625\end{array}$ & $\begin{array}{l}\text { Yes (eggs, larvae and/ } \\
\text { or metamorphs present) }\end{array}$ & $619 \mathrm{~m}$; ponds on river side; mixed deciduous forest \\
\hline & $\begin{array}{l}\text { N45 } 17.190 \\
\text { E24 } 14.102\end{array}$ & $\begin{array}{l}\text { Inaparent (adults and/ } \\
\text { or subadults only) }\end{array}$ & $689 \mathrm{~m}$; road-side ditch; deciduous forest, mainly beech \\
\hline & $\begin{array}{l}\text { N45 } 17.483 \\
\text { E24 } 14.968\end{array}$ & $\begin{array}{l}\text { Inaparent (adults and/ } \\
\text { or subadults only) }\end{array}$ & $757 \mathrm{~m}$; road-side ditch; deciduous forest, mainly beech \\
\hline \multirow[t]{5}{*}{ Păușa valley 2014-16 } & $\begin{array}{ll}\text { N45 } & 16.127 \\
\text { E24 } 19.894\end{array}$ & $\begin{array}{l}\text { Inaparent (adults and/ } \\
\text { or subadults only) }\end{array}$ & $335 \mathrm{~m}$; stream in mixed deciduous forest \\
\hline & $\begin{array}{l}\text { N45 } 16.551 \\
\text { E24 } 20.123 \\
\end{array}$ & $\begin{array}{l}\text { Inaparent (adults and/ } \\
\text { or subadults only) }\end{array}$ & $378 \mathrm{~m}$; stream in mixed deciduous forest \\
\hline & $\begin{array}{ll}\text { N45 } & 16.671 \\
\text { E24 } 20.145 \\
\end{array}$ & $\begin{array}{l}\text { Inaparent (adults and/ } \\
\text { or subadults only) }\end{array}$ & $392 \mathrm{~m}$; stream in mixed deciduous forest \\
\hline & $\begin{array}{l}\text { N45 } 16.841 \\
\text { E24 } 20.100 \\
\end{array}$ & $\begin{array}{l}\text { Yes (eggs, larvae and/ } \\
\text { or metamorphs present) }\end{array}$ & $\begin{array}{l}409 \mathrm{~m} \text {; ponds on stream side, tail of dam } \\
\text { lake in mixed deciduous forest }\end{array}$ \\
\hline & $\begin{array}{l}\text { N45 } 17.172 \\
\text { E24 } 19.903 \\
\end{array}$ & $\begin{array}{l}\text { Inaparent (adults and/ } \\
\text { or subadults only) }\end{array}$ & $447 \mathrm{~m}$; road-side ditch; mixed deciduous forest \\
\hline $\begin{array}{l}\text { Stânișoara } \\
\text { monastery } 2016\end{array}$ & \begin{tabular}{|l|l|} 
N45 18.098 \\
E24 20.371
\end{tabular} & $\begin{array}{l}\text { Yes (eggs, larvae and/ } \\
\text { or metamorphs present) }\end{array}$ & $736 \mathrm{~m}$; spring ponds; grassy clearing \\
\hline $\begin{array}{l}\text { Căciulata valley } \\
2014-16\end{array}$ & $\begin{array}{l}\text { N45 } 15.478 \\
\text { E24 } 18.689 \\
\end{array}$ & $\begin{array}{l}\text { Yes (eggs, larvae and/ } \\
\text { or metamorphs present) }\end{array}$ & $\begin{array}{l}377 \mathrm{~m} \text {; puddles in road and stream } \\
\text { in mixed deciduous forest }\end{array}$ \\
\hline \multirow[t]{5}{*}{ Olt valley $2014-16$} & $\begin{array}{ll}\text { N45 } & 20.341 \\
\text { E24 } & 16.764 \\
\end{array}$ & $\begin{array}{l}\text { Inaparent (adults and/ } \\
\text { or subadults only) }\end{array}$ & $\begin{array}{l}331 \mathrm{~m} \text {; road-side ditch; mixed } \\
\text { deciduous forest; dried } 2015\end{array}$ \\
\hline & $\begin{array}{l}\text { N45 } 23.036 \\
\text { E24 } 18.022 \\
\end{array}$ & $\begin{array}{l}\text { Inaparent (adults and/ } \\
\text { or subadults only) }\end{array}$ & $\begin{array}{l}358 \mathrm{~m} \text {; road-side spring pond; mixed } \\
\text { deciduous forest; dried } 2015\end{array}$ \\
\hline & $\begin{array}{ll}\text { N45 } & 20.171 \\
\text { E24 } & 16.801 \\
\end{array}$ & $\begin{array}{l}\text { Inaparent (adults and/ } \\
\text { or subadults only) }\end{array}$ & $280 \mathrm{~m}$; stream in rural mosaic \\
\hline & $\begin{array}{l}\text { N45 } 22.424 \\
\text { E24 } 18.042 \\
\end{array}$ & $\begin{array}{l}\text { Inaparent (adults and/ } \\
\text { or subadults only) }\end{array}$ & $\begin{array}{l}314 \mathrm{~m} \text {; ponds on river side; mixed } \\
\text { deciduous forest and grassy clearing }\end{array}$ \\
\hline & $\begin{array}{l}\text { N45 } 20.516 \\
\text { E24 } 16.834 \\
\end{array}$ & $\begin{array}{l}\text { Inaparent (adults and/ } \\
\text { or subadults only) }\end{array}$ & $307 \mathrm{~m}$; large roadside pond; mixed deciduous forest \\
\hline \multirow[t]{4}{*}{$\begin{array}{l}\text { Lotrișor (Căpățânii) } \\
\text { 2014-16 }\end{array}$} & $\begin{array}{l}\text { N45 } 23.597 \\
\text { E24 } 16.376\end{array}$ & $\begin{array}{l}\text { Inaparent (adults and/ } \\
\text { or subadults only) }\end{array}$ & $\begin{array}{l}440 \mathrm{~m} \text {; ponds on stream side; mixed } \\
\text { deciduous forest and grassy clearing }\end{array}$ \\
\hline & $\begin{array}{l}\text { N45 } 24.243 \\
\text { E24 } 14.793\end{array}$ & $\begin{array}{l}\text { Inaparent (adults and/ } \\
\text { or subadults only) }\end{array}$ & $\begin{array}{l}526 \mathrm{~m} \text {; road-side ditch; mixed } \\
\text { deciduous forest; dried } 2015\end{array}$ \\
\hline & $\begin{array}{l}\text { N45 } 24.069 \\
\text { E24 } 14.940\end{array}$ & $\begin{array}{l}\text { Yes (eggs, larvae and/ } \\
\text { or metamorphs present) }\end{array}$ & $507 \mathrm{~m}$; ponds on stream side; disturbed gravel bed \\
\hline & $\begin{array}{l}\text { N45 } 23.839 \\
\text { E24 } 15.548 \\
\end{array}$ & $\begin{array}{l}\text { Yes (eggs, larvae and/ } \\
\text { or metamorphs present) }\end{array}$ & $\begin{array}{l}424 \mathrm{~m} \text {; ponds on stream side; mixed } \\
\text { deciduous forest and grassy clearing }\end{array}$ \\
\hline Băiașu valley 2014-16 & $\begin{array}{l}\text { N45 } 22.164 \\
\text { E24 } 20.841\end{array}$ & $\begin{array}{l}\text { Inaparent (adults and/ } \\
\text { or subadults only) }\end{array}$ & $\begin{array}{l}357 \mathrm{~m} \text {; large backwater stream-side pond, mixed } \\
\text { deciduous forest and grassy clearing; formed } 2015\end{array}$ \\
\hline
\end{tabular}


Table 3 (continued)

\begin{tabular}{|c|c|c|c|}
\hline $\begin{array}{l}\text { Locality/population, } \\
\text { visit year(s) }\end{array}$ & $\begin{array}{l}\text { GPS } \\
\text { Coordinates }\end{array}$ & Reproduction & Altitude and ecological characteristics \\
\hline & $\begin{array}{l}\text { N45 } 22.200 \\
\text { E24 21.575 }\end{array}$ & $\begin{array}{l}\text { Yes (eggs, larvae and/ } \\
\text { or metamorphs present) }\end{array}$ & $\begin{array}{l}417 \mathrm{~m} \text {; road-side ditch, mixed deciduous } \\
\text { forest and grassy clearing; formed } 2016\end{array}$ \\
\hline \multirow[t]{11}{*}{$\begin{array}{l}\text { Pripoare-Dăngești } \\
\text { 2015-16 }\end{array}$} & $\begin{array}{l}\text { N45 } 19.577 \\
\text { E24 25.394 }\end{array}$ & $\begin{array}{l}\text { Inaparent (adults and/ } \\
\text { or subadults only) }\end{array}$ & $\begin{array}{l}627 \mathrm{~m} \text {; road-side ditch, mixed beech and } \\
\text { coniferous forest; drained } 2016\end{array}$ \\
\hline & $\begin{array}{l}\text { N45 } 18.365 \\
\text { E24 } 25.106\end{array}$ & $\begin{array}{l}\text { Yes (eggs, larvae and/ } \\
\text { or metamorphs present) }\end{array}$ & $675 \mathrm{~m}$; spring ponds, pasture \\
\hline & $\begin{array}{l}\text { N45 } 18.604 \\
\text { E24 } 25.400\end{array}$ & $\begin{array}{l}\text { Yes (eggs, larvae and/ } \\
\text { or metamorphs present) }\end{array}$ & $\begin{array}{l}702 \mathrm{~m} \text {; trough for watering cattle, mixed } \\
\text { beech and coniferous forest }\end{array}$ \\
\hline & $\begin{array}{ll}\text { N45 } 19.212 \\
\text { E24 } 25.489\end{array}$ & $\begin{array}{l}\text { Inaparent (adults and/ } \\
\text { or subadults only) }\end{array}$ & $\begin{array}{l}719 \mathrm{~m} \text {; ponds on stream side, mixed } \\
\text { beech and coniferous forest }\end{array}$ \\
\hline & $\begin{array}{l}\text { N45 } 19.484 \\
\text { E24 25.389 }\end{array}$ & $\begin{array}{l}\text { Yes (eggs, larvae and/ } \\
\text { or metamorphs present) }\end{array}$ & $\begin{array}{l}694 \mathrm{~m} \text {; ponds on stream side, mixed } \\
\text { beech and coniferous forest }\end{array}$ \\
\hline & $\begin{array}{l}\text { N45 } 20.269 \\
\text { E24 } 25.128\end{array}$ & $\begin{array}{l}\text { Inaparent (adults and/ } \\
\text { or subadults only) }\end{array}$ & $\begin{array}{l}611 \mathrm{~m} \text {; road-side ditch, mixed beech and } \\
\text { coniferous forest; drained } 2016\end{array}$ \\
\hline & $\begin{array}{l}\text { N45 } 20.442 \\
\text { E24 } 24.996\end{array}$ & $\begin{array}{l}\text { Yes (eggs, larvae and/ } \\
\text { or metamorphs present) }\end{array}$ & $\begin{array}{l}597 \mathrm{~m} \text {; road-side ditch, mixed beech and } \\
\text { coniferous forest, grassy clearing; drained } 2016\end{array}$ \\
\hline & $\begin{array}{l}\text { N45 } 20.605 \\
\text { E24 } 25.104\end{array}$ & $\begin{array}{l}\text { Yes (eggs, larvae and/ } \\
\text { or metamorphs present) }\end{array}$ & $\begin{array}{l}591 \mathrm{~m} \text {; ponds on stream side, mixed beech } \\
\text { and coniferous forest; drained } 2016\end{array}$ \\
\hline & $\begin{array}{l}\text { N45 } 18.956 \\
\text { E24 } 25.398\end{array}$ & $\begin{array}{l}\text { Yes (eggs, larvae and/ } \\
\text { or metamorphs present) }\end{array}$ & $\begin{array}{l}727 \mathrm{~m} \text {; road-side ditch, mixed beech and } \\
\text { coniferous forest, grassy clearing }\end{array}$ \\
\hline & $\begin{array}{l}\text { N45 } 18.495 \\
\text { E24 } 25.370\end{array}$ & $\begin{array}{l}\text { Yes (eggs, larvae and/ } \\
\text { or metamorphs present) }\end{array}$ & $664 \mathrm{~m}$; ponds in pasture, at edge of mixed forest \\
\hline & $\begin{array}{l}\text { N45 } 19.892 \\
\text { E24 25.377 }\end{array}$ & $\begin{array}{l}\text { Yes (eggs, larvae and/ } \\
\text { or metamorphs present) }\end{array}$ & $\begin{array}{l}660 \mathrm{~m} \text {; ponds on stream side, mixed } \\
\text { beech and coniferous forest }\end{array}$ \\
\hline \multirow[t]{5}{*}{$\begin{array}{l}\text { Pătești (Sălătruc) } \\
\text { valley } 2016\end{array}$} & $\begin{array}{l}\text { N45 } 16.936 \\
\text { E24 } 23.039\end{array}$ & $\begin{array}{l}\text { Yes (eggs, larvae and/ } \\
\text { or metamorphs present) }\end{array}$ & $553 \mathrm{~m}$; spring pond in pasture \\
\hline & $\begin{array}{l}\text { N45 } 17.005 \\
\text { E24 } 22.922\end{array}$ & $\begin{array}{l}\text { Yes (eggs, larvae and/ } \\
\text { or metamorphs present) }\end{array}$ & $553 \mathrm{~m}$; pond in small landslide in clearing \\
\hline & $\begin{array}{l}\text { N45 } 17.555 \\
\text { E24 } 22.506\end{array}$ & $\begin{array}{l}\text { Yes (eggs, larvae and/ } \\
\text { or metamorphs present) }\end{array}$ & $\begin{array}{l}645 \mathrm{~m} \text {; road-side ditch, mixed beech and } \\
\text { coniferous forest, grassy clearing }\end{array}$ \\
\hline & $\begin{array}{l}\text { N45 } 17.860 \\
\text { E24 } 22.465\end{array}$ & $\begin{array}{l}\text { Inaparent (adults and/ } \\
\text { or subadults only) }\end{array}$ & $\begin{array}{l}687 \mathrm{~m} \text {; road-side ditch, mixed } \\
\text { beech and coniferous forest }\end{array}$ \\
\hline & $\begin{array}{l}\text { N45 } 18.129 \\
\text { E24 } 22.261\end{array}$ & $\begin{array}{l}\text { Inaparent (adults and/ } \\
\text { or subadults only) }\end{array}$ & $\begin{array}{l}723 \mathrm{~m} \text {; road-side ditch, mixed } \\
\text { beech and coniferous forest }\end{array}$ \\
\hline \multirow[t]{3}{*}{$\begin{array}{l}\text { Lotrişor (Cozia) } \\
\text { 2014-16 }\end{array}$} & $\begin{array}{l}\text { N45 } 22.242 \\
\text { E24 } 18.527\end{array}$ & $\begin{array}{l}\text { Inaparent (adults and/ } \\
\text { or subadults only) }\end{array}$ & $\begin{array}{l}373 \mathrm{~m} \text {; road-side ditch; mixed } \\
\text { deciduous forest; formed } 2015\end{array}$ \\
\hline & $\begin{array}{l}\text { N45 } 22.143 \\
\text { E24 } 18.668\end{array}$ & $\begin{array}{l}\text { Inaparent (adults and/ } \\
\text { or subadults only) }\end{array}$ & $383 \mathrm{~m}$; road-side ditch; mixed deciduous forest \\
\hline & $\begin{array}{l}\text { N45 } 21.821 \\
\text { E24 } 19.079\end{array}$ & $\begin{array}{l}\text { Yes (eggs, larvae and/ } \\
\text { or metamorphs present) }\end{array}$ & $418 \mathrm{~m}$; road-side ditch; mixed deciduous forest \\
\hline $\begin{array}{l}\text { Near "La Leurde", } \\
\text { Pleașa ridge } 2015\end{array}$ & $\begin{array}{l}\text { N45 } 19.344 \\
\text { E24 } 22.287 \\
\end{array}$ & $\begin{array}{l}\text { Inaparent (adults and/ } \\
\text { or subadults only) }\end{array}$ & $\begin{array}{l}1115 \mathrm{~m} \text {; road-side ditch, mixed } \\
\text { beech and coniferous forest }\end{array}$ \\
\hline \multirow[t]{2}{*}{ Doabra valley 2016} & $\begin{array}{l}\text { N45 } 20.939 \\
\text { E24 } 14.638\end{array}$ & $\begin{array}{l}\text { Inaparent (adults and/ } \\
\text { or subadults only) }\end{array}$ & $330 \mathrm{~m}$; stream in rural mosaic \\
\hline & $\begin{array}{l}\text { N45 } 21.002 \\
\text { E24 } 14.617\end{array}$ & $\begin{array}{l}\text { Inaparent (adults and/ } \\
\text { or subadults only) }\end{array}$ & $\begin{array}{l}337 \mathrm{~m} \text {; road-side ditch, mixed deciduous } \\
\text { forest, grassy clearing }\end{array}$ \\
\hline \multirow[t]{3}{*}{ Vasilatu valley 2016} & $\begin{array}{l}\text { N45 } 21.096 \\
\text { E24 } 12.628\end{array}$ & $\begin{array}{l}\text { Inaparent (adults and/ } \\
\text { or subadults only) }\end{array}$ & $\begin{array}{l}358 \mathrm{~m} \text {; road-side ditch, mixed deciduous } \\
\text { forest, grassy clearing }\end{array}$ \\
\hline & $\begin{array}{l}\text { N45 } 22.067 \\
\text { E24 } 12.486\end{array}$ & $\begin{array}{l}\text { Yes (eggs, larvae and/ } \\
\text { or metamorphs present) }\end{array}$ & $\begin{array}{l}401 \mathrm{~m} \text {; road-side ditch, mixed deciduous } \\
\text { forest, grassy clearing }\end{array}$ \\
\hline & $\begin{array}{l}\text { N45 } 22.568 \\
\text { E24 } 12.570\end{array}$ & $\begin{array}{l}\text { Inaparent (adults and/ } \\
\text { or subadults only) }\end{array}$ & $\begin{array}{l}421 \mathrm{~m} \text {; road-side ditch, mixed deciduous } \\
\text { forest, grassy clearing }\end{array}$ \\
\hline
\end{tabular}




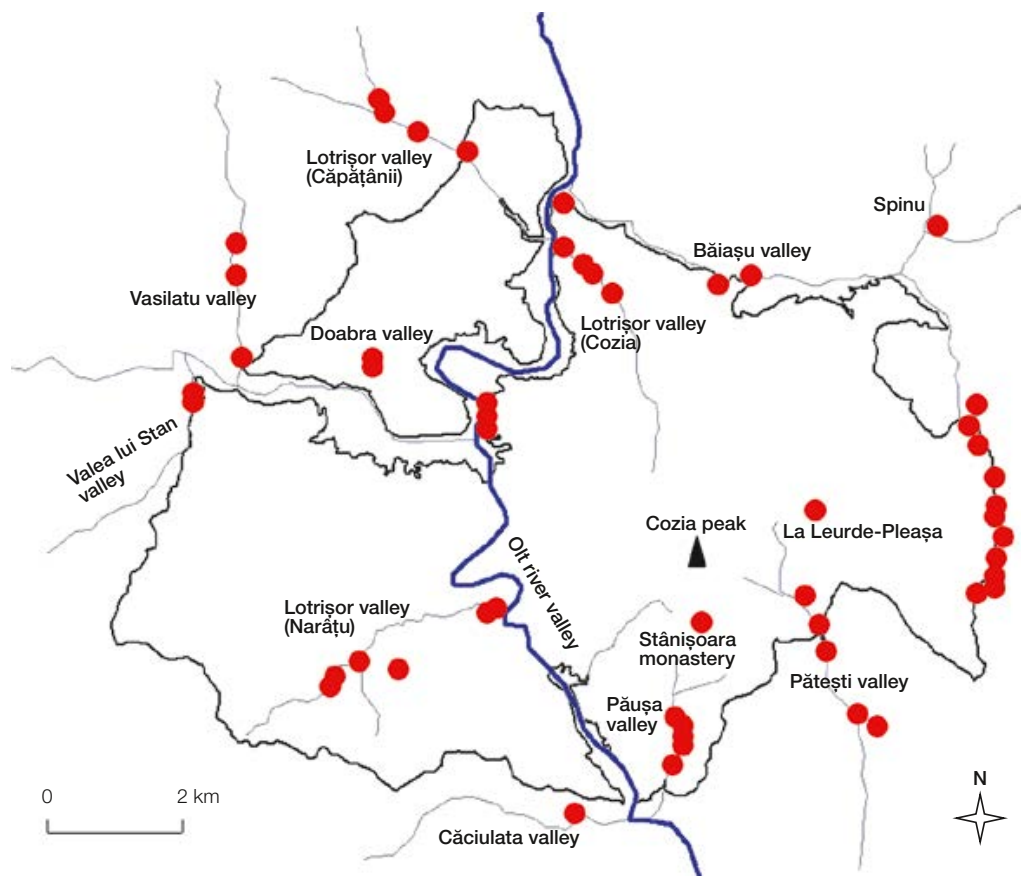

Fig. 1 - Map showing the distribution of Bombina variegata (in red) in and around Cozia National Park (Park limits after Ministry of Environment, Water and Forests data, downloaded from http://www. mmediu.ro/articol/date-gis/434).

nothing of the sort. Clearly major floods are a disturbing factor, but Bombina variegata populations are quickly back - either from locally surviving individuals (more likely, since they were breeding adults) or from dispersion/colonization.

Human impact is manifest in both the destruction and the creation of transient habitats. Along the road from Pripoare to Dăngești, a series of road-side ditches (obviously man-made, in relation to the road) were hosting a rich Bombina variegata population up to 2015; many were drained till the spring of 2016 by road improvement works, but new water pits were created in the same process. Also, in the same area, troughs for watering livestock provide a good habitat, stable on the long term, where Bombina variegata breeds successfully. On the whole, Bombina variegata can be

Average character score for each locality/population.

Table 4

\begin{tabular}{|l|l|l|}
\hline Population & No. of specimens examined & $\begin{array}{l}\text { Average morphological score } \\
\text { - B. variegata vs. B. bombina }\end{array}$ \\
\hline Valea lui Stan & 53 & 8.75 \\
\hline Lotrișor (Narâțu) & 45 & 7.5 \\
\hline Spinu & 63 & 9 \\
\hline Păușa & 77 & 8 \\
\hline Căciulata & 48 & 8.5 \\
\hline Lotrișor (Căpățânii) & 44 & 8 \\
\hline Valea Oltului & 10 & 8.4 \\
\hline
\end{tabular}




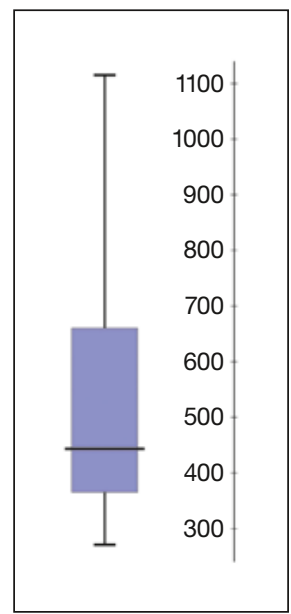

Fig. 2 - Boxplot (showing minimum and maximum, quartiles and median) of the altitudinal distribution of Bombina variegata finding points.

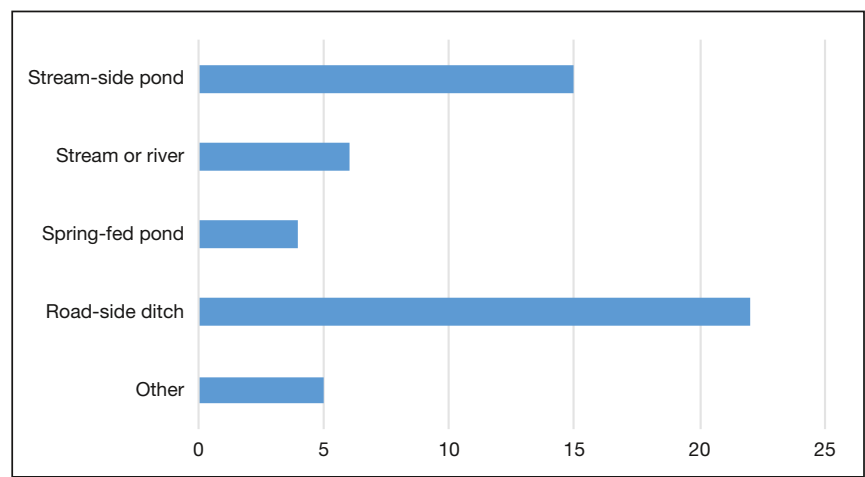

Fig. 3 - Bar diagram of the grouping of the Bombina variegata finding points by habitat type.

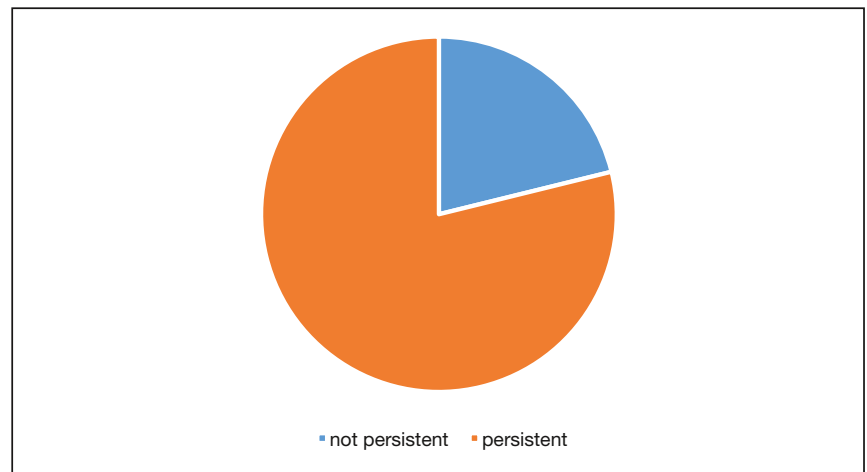

Fig. 4 - Pie diagram of habitat persistence among Bombina variegata habitats investigated in more than one year. 
said to "hop" from habitat to habitat and from year to year, the metapopulational dynamic, evidenced by the turnover of microhabitats, being apparently quite intense; it readily occupies man-made habitats. However, in intensely human-altered habitats (e.g. on Păușa, Doabra and other valleys) Bombina variegata appears at or beyond the edge of human habitation along the water course; in the villages per se it was never found, and only twice in "rural mosaic" habitat (hayfields, orchards etc., with close proximity of houses) surrounding the water body (Tab. 3).

Interestingly, not all microhabitats where Bombina variegata was seen apparently harboured successful reproduction - on the whole more than half did not (Figs 5,6). In those we could not see any eggs, tadpoles and metamorphs that would indicate reproduction in the place and not immigration from somewhere else. Of course, this does not mean that in those areas reproduction does not take place at all, but, however, it does indicate lower success and lower recruitment in these areas as compared to places where the successful reproduction is obvious from the large number of larvae and metamorphs. On the Olt valley all points where Bombina variegata was found (small ponds more or less distant from the Olt river itself) appeared devoid of successful reproductive activity (Tab. 3). On other valleys, reproductive activity appears to be concentrated in one or a few favourable spots. A good example of this is Păușa

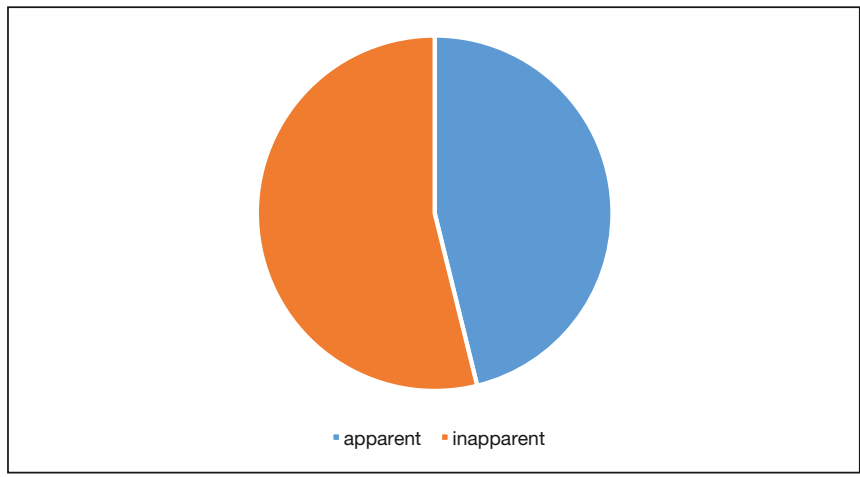

Fig. 5 - Pie diagram of apparent versus inapparent successful reproduction in investigated Bombina variegata habitats.

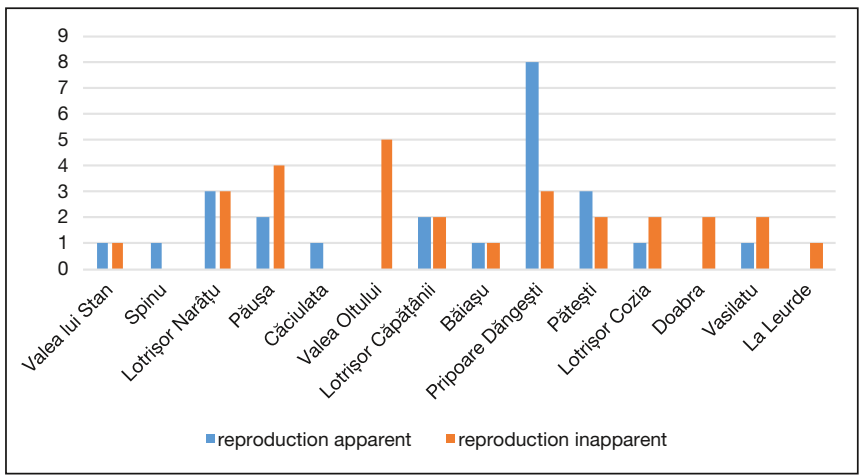

Fig. 6 - Bar diagram of apparent versus inapparent successful reproduction in investigated Bombina variegata habitats per population/area. 
valley, where in only one place successful reproduction was observed; in all others adults and subadults were seen - which may come from the one reproduction point observed, or from others not detected by us (however, we consider the probability for this to be scarce); or, alternatively, in other years good conditions for breeding may appear in other spots and be exploited. We can, however, note that on the narrow, rocky, relatively steep valleys closer to the Olt valley (and to its affluent the Lotru) good breeding habitats are less common than in the rolling-hill habitats (e.g. SpinuPripoare-Dăngești) - fig. 6.

As previously noted (Iftime \& Iftime, 2007), the Bombina variegata populations from Cozia show traces of past introgressive hybridization with Bombina bombina. The scores obtained (Tab. 4, Fig. 7) would place all populations in the "pure" Bombina variegata or B. variegata-like hybrid cathegory according to the quoted methodology; however, as also noted by us previously (Iftime \& Iftime, 2007) there are individuals with lower B. variegata score (and conversely higher B. bombina score), i.e. around 5, which fall around the typical value of first-generation hybrids (Fig. 7). These reinforce our hypothesis that there was an introgressive hybridization in the past (as it still happens in some foothills of the Southern Carpathians - Iftime \& Iftime, 2011). The penetration channel for B. bombina and associated gene flow was, likely, along the

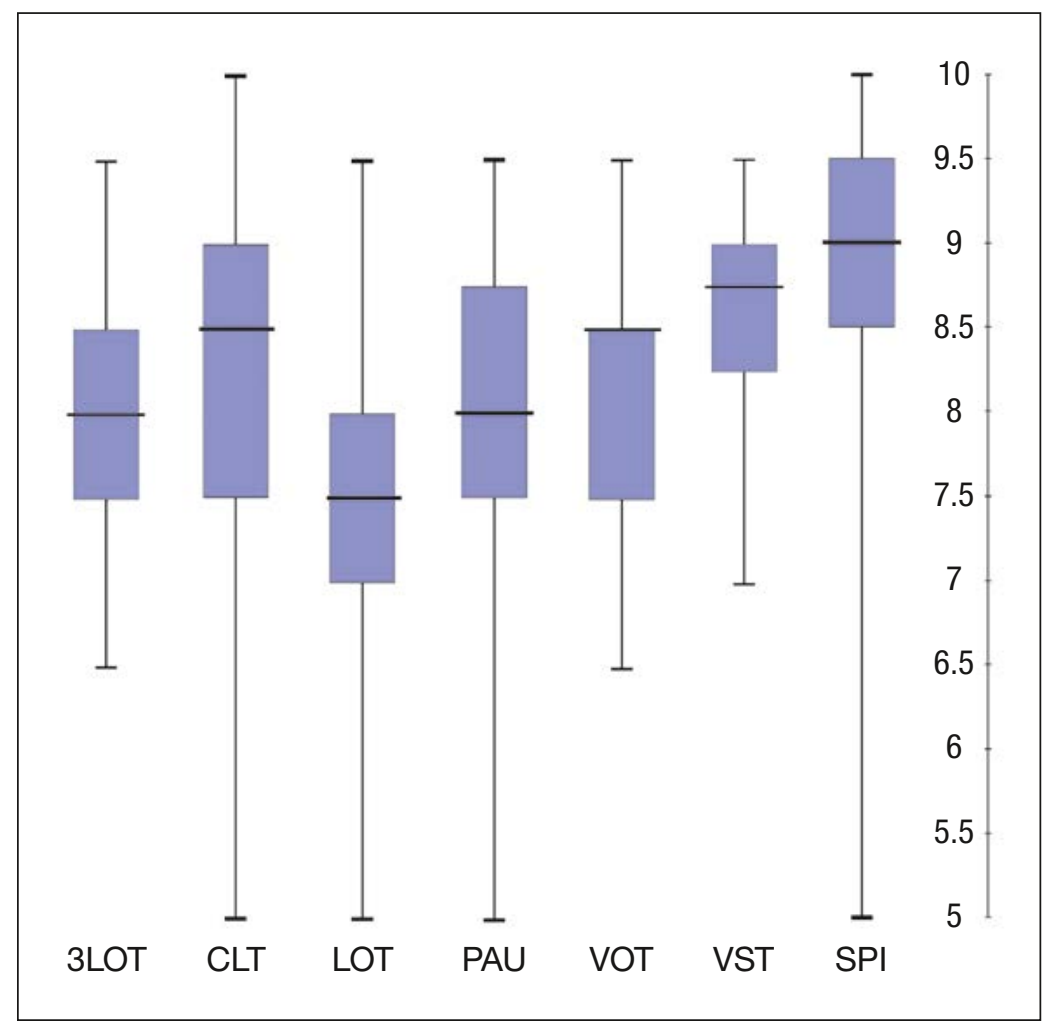

Fig. 7 - Boxplots (showing minimum and maximum, quartiles and median) of the Bombina variegata vs. Bombina bombina morphological score for populations investigated in this respect. Abbreviations: 3LOT = Lotrișor-Căpățânii; CLT = Căciulata; LOT = Lotrișor-Narâțu; PAU = Păușa; VOT = Olt valley; VST $=$ Valea lui Stan; SPI $=$ Spinu. 
Olt valley before its damming (Iftime \& Iftime, 2007) which is further supported by the higher $B$. variegata score of the populations located further away from the Olt valley (Fig. 7). We may also note that the hybrid score data from Iftime \& Iftime, 2007, which were obtained on Păușa valley, were lower than the corresponding current scores: the average was 7.22 in 2007 vs. 8 currently, while the minimum was 4.5 vs. 5 currently. This small increase may pertain to a larger sampling (but one should notice the increase in the minimum), be stochastic, or, alternatively, it may indicate selective forces acting against lower hybrid fitness.

On the whole, the Bombina variegata populations in the Cozia National Park are widespread, quite resilient to the current level of natural and human disturbance, moderately anthropophilic (i.e. extensively utilising man-made habitats, but not occuring in dense human settlements) and showing a pattern of previous introgressive hybridization with $B$. bombina. Their habitats show different quality, connectivity and dynamics according to the local topography; whether there is genuine fragmentation or not should be ascertained by more detailed means of investigation. These data should be useful for the conservation-oriented management of the local population of Bombina variegata within the National Park and Natura 2000 site context.

\section{ACKNOWLEDGEMENTS}

This work was funded by Romanian UEFISCDI Executive Unit for Financing Education Higher Research Development and Innovation, Grant No. 113/2014, code: PN-II-PT-PCCA-2013-4-1489.

\section{REFERENCES}

COGĂLNICEANU, D. (1997) Practicum de ecologie a amfibienilor: Metode şi tehnici în studiul ecologiei amfibienilor. Edit. Universităţii din Bucureşti, 122 pp. (in Romanian)

COGĂLNICEANU, D, F. AIOANEI, B. MATEI (2000) Amfibienii din România. Determinator. Edit. Ars Docendi, Bucureşti, 100 pp. (in Romanian)

COGĂLNICEANU, D., P. SZÉKELY, C. SAMOILĂ, R. IOSIF, M. TUDOR, R. PLĂIAŞU, F. STĂNESCU, L. ROZYLOWICZ (2013a) Diversity and distribution of amphibians in Romania. ZooKeys, 296: 35-57.

COGĂLNICEANU, D., L. ROZYLOWICZ, P. SZÉKELY, C. SAMOILĂ, F. STĂNESCU, M. TUDOR, D. SZÉKELY, R. IOSIF (2013b) Diversity and distribution of reptiles in Romania. ZooKeys, 341: 49-76.

COVACIU-MARCOV, S. D., I. SAS, D. CADLET,, V. PETER, B. ANTAL (2003) Research about the hybridization area between Bombina bombina and Bombina variegata of the middle course of Barcău river (Bihor county, Romania). Analele Universităţii din Oradea, Fascicula Biologie, 10: 65-79.

FUHN, I. (1960) Amphibia. In: Fauna R.P.R., 14, 1, Edit. Academiei R.S.R., Bucureşti, 288 pp. (in Romanian)

GHIRA, I., G. MARA (2000). Using the allelomorphic feature in identifying two species belonging to genus Bombina (Anura, Discoglossidae) from Transylvania. Studia Universitatis BabesBolyai, Cluj-Napoca, 25: 85-95.

GHIRA, I., M. VENCZEL, S. COVACIU-MARCOV, G. MARA, P. GHILE, T. HARTEL, ZS. TÖRÖK, L. FARKAS, T. RÁCZ, Z. FARKAS, T. BRAD (2002) Mapping of Transsylvanian Herpetofauna. Nymphaea - Folia naturae Bihariae, 29: 145-201.

GOLLMANN, G. (1984) Allozymic and morphological variation in the hybrid zone between Bombina bombina and Bombina variegata (Anura, Discoglossidae), in northeastern Austria. Zeitschrift für Zoologische Systematik und Evolutionsforschung, 22: 51-64.

IFTIME A. (2005) Amphibia. Reptilia. In: N. Botnariuc, V. Tatole, (eds.) Cartea Roşie a vertebratelor României, Ed. Curtea Veche, Bucureşti, pp. 173-214. (in Romanian)

IFTIME, A., O. IFTIME (2006) Preliminary data on the herpetofauna of the Cozia massif (Romania). 1. Reptiles. Travaux du Muséum National d'Histoire Naturelle "Grigore Antipa", 49: 331-340.

IFTIME, A., O. IFTIME (2007) Preliminary data on the herpetofauna of the Cozia massif(Romania). 2. Amphibians. Travaux du Muséum National d'Histoire Naturelle "Grigore Antipa”, 50: 259-271. 
IFTIME, A., O. IFTIME (2011) Note on the herpetofauna of the Vâlcan mountains and their foothills (Southern Carpathians, Romania). Travaux du Muséum National d'Histoire Naturelle "Grigore Antipa", 54(2): 513-521.

IFTIME, A., O. IFTIME (2014) Note on the amphibians and reptiles of the "Nordul Gorjului de Est" site of community interest and adjacent areas (Southern Carpathians, Romania). North-Western Journal of Zoology, 10(1): S44-S50

SAS, I., S.-D. COVACIU-MARCOV, M. POP, R.-D. ILE, N. SZEIBEL, C. DUMA (2005) About a closed hybrid population between Bombina bombina and Bombina variegata from Oradea (Bihor county, Romania). North-Western Journal of Zoology, 1: 41-60.

SPEYBROECK, J., W. BEUKEMA, B. BOK, J. VAN DER VOORT, I. VELIKOV (2016) Field Guide to the Amphibians and Reptiles of Britain and Europe (British Wildlife Field Guides). Bloomsbury Natural History, London/New York, $432 \mathrm{pp}$.

STUGREN, B., (1980) Geographical variation of the fire-bellied toad (Bombina bombina (L.) in the USSR. (Amphibia, Anura, Discoglossidae). Zoologische Abhandlungen/Staatliches Museum für Tierkunde Dresden, 36 (5): 101-115.

SZYMURA, J. M., N. H. BARTON (1991) The genetic structure of the hybrid zone between the firebellied toads Bombina bombina and Bombina variegata: comparisons between transects and between loci. Evolution 45(2): 237-261. 